\title{
Different Meta-Analysis Methods, Different Answers: The Case of Exercise for Older Acute Medical Patients
}

\author{
Natalie A. de Morton ${ }^{*}, 1$ and Jennifer L. Keating ${ }^{2}$ \\ ${ }^{I}$ School of Primary Health Care, Department of General Practice, Faculty of Medicine, Nursing and Health Sciences, \\ Monash University, Building 1, 270 Ferntree Gully Road, Notting Hill, Victoria 3168, Australia \\ ${ }^{2}$ School of Primary Health Care, Department of Physiotherapy, Faculty of Medicine, Nursing and Health Sciences, \\ Monash University - Peninsula Campus, PO Box 527, Frankston, 3199, Victoria, Australia
}

\begin{abstract}
The aim of this study was to illustrate the potential for confounding when interpreting group mean data from systematic reviews for a heterogeneous participant population. A case report comparing the results obtained from a Cochrane review and meta-analysis compared to an individual patient data (IPD) meta-analysis was conducted. Participants were 396 older patients admitted to a general medical ward at two acute public hospitals. For the intervention group, an exercise program was provided for 20-30 minutes twice per day in addition to usual care. The control group received usual hospital care. The primary outcome measure was changed in Barthel Index scores between hospital admission and discharge. Meta-analysis of group mean data provided evidence that additional exercise for older medical patients does not significantly improve Barthel Index scores at hospital discharge (fixed effects model, 0.17 [-0.06 to 0.40$] \mathrm{I}^{2}=0 \%$ ). Results of IPD meta-analysis indicated that additional exercise significantly improved discharge Barthel Index scores for patients who required assistance to ambulate at hospital admission (coefficient for group 5.4 [1.38 to 9.40], p=0.01) but not for those who were non ambulant or independently ambulant at hospital admission. This case report provides an example where performing meta-analysis using group mean data for heterogeneous populations can result in effective interventions being discarded.
\end{abstract}

Keywords: Systematic review, meta-analysis, aged people, exercise, statistical method.

\section{INTRODUCTION}

Systematic review provides a method for addressing a research question by systematically appraising, synthesising and evaluating scientific literature. Systematic reviews are important for providing an evidence base that informs clinical practice and identifying areas of healthcare that require further research. However, there is a potential for heterogeneity in person response to an intervention to confound interpretation of group mean data. Heterogeneity may be amplified when a diagnosis or classification includes a broad spectrum or mixture of different health conditions. One approach is to narrow participant inclusion criteria, but for many patient groups this is not a possible way in which the inclusion criteria reduces the heterogeneity which varies across relevant studies.

For heterogeneous populations, consideration of differential intervention effects within the population, may enhance a view of the intervention utility. Individual patient data (IPD) meta-analysis has been referred to as the "gold standard" for systematic reviews [1], as it offers a method that facilitates detailed subgroup analysis using patient characteristics level. IPD meta-analysis allows individual patient characteristics to be included as covariates and patients who are the most or

*Address correspondence to this author at the Department of General Practice, School of Primary Health Care, Faculty of Medicine, Nursing and Health Sciences, Monash University, Building 1, 270 Ferntree Gully Road, Notting Hill, Victoria 3168, Australia;

E-mail: natalie.demorton@med.monash.edu.au the least responsive to an intervention can be identified. It is possible for heterogeneous patient populations that differing conclusions regarding intervention effectiveness could be obtained using meta-analysis of group mean data compared to IPD meta-analysis.

The advantages of IPD meta-analysis compared to group mean data meta-analysis have been well documented in the literature. For example, Berlin et al. [2] and Lambert et al. [3] have recommended that IPD meta-analysis should be used whenever possible to explore individual patient characteristics or heterogeneity. However, Tudur Smith et al. [4] have more specifically argued that comparisons of results obtained using group mean aggregate or IPD meta-analysis are required to identify the conditions, where the IPD metaanalysis may be most beneficial.

This report illustrates a case where there were advantages of meta-analysing using IPD compared to group mean aggregate data for a participant population, who were heterogenous with respect to the severity of the condition.

\section{METHOD}

A recent Cochrane review [5, 6] identified randomised controlled trials and controlled clinical trials that investigated the effect of additional exercise for older medical patients ( $>65$ years) compared to usual care during acute hospitalisation. Group means change in activity limitation scores on the Barthel Index (score range 0-100) between acute hospital admission and discharge from two trials which were 
pooled in meta-analysis. Both trials were conducted in acute public hospitals in Melbourne, Australia.

Older general medical patients are heterogeneous patient population (e.g. they have a diverse range of medical conditions, co-morbidities, prognoses, physical abilities). Patient's response to an additional exercise program during hospitalisation was considered likely to be highly variable across participants. An IPD meta-analysis was therefore conducted [7] according to the methodological guidelines provided by the Cochrane Collaboration Working Group on IPD Metaanalysis [8]. All randomised patient data were included with the intention to treat analysis performed. The effect of the intervention on discharge Barthel Index (BI) scores was analysed using multiple linear regression modelling.

\section{RESULTS}

In the Cochrane meta-analysis, small effect sizes were identified in pooled change in BI scores that favoured the intervention group but were not statistically significant. The trial by de Morton et al. (2006) [9] reported an effect size of $0.15(95 \% \mathrm{CI}-0.16$ to 0.45$)$ and the trial by Jones et al. (2006) [10] reported an effect size of $0.20(95 \% \mathrm{CI}-0.15$ to 0.55 ). Meta-analysis of group mean data indicated no effect of additional exercise intervention for older medical patients on change in BI scores between acute hospital admission and discharge (fixed effects model, 0.17 [-0.06 to 0.40]). Significant statistical heterogeneity was not identified $\left(\mathrm{p}=0.81, \mathrm{I}^{2}=\right.$ $0 \%)$.

In contrast, results of IPD meta-analysis indicated that additional exercise significantly improved discharge Barthel Index scores for patients who required assistance to ambulate at hospital admission (coefficient for group 5.4 [1.38 to 9.40], $\mathrm{p}=0.01$ ), but not for those who were non ambulant (coefficient $2.86,-8.66$ to $14.39, \mathrm{p}=0.61$ ) or independently ambulant (coefficient $-1.74,-4.71$ to $1.24, \mathrm{p}=0.25$ ) at hospital admission. This is a biologically plausible finding as the patients in a better condition stand to gain little from a short exercise program and those not in a good condition are less likely to participate.

\section{DISCUSSION}

Differing results were obtained using group mean aggregate data compared to IPD in these two approaches to metaanalysis. Results of meta-analysis from the Cochrane review indicated no effect of additional exercise for older acute general medical patients. In contrast, IPD meta-analysis identified a significant interventional effect for patients who required assistance or supervision to ambulate at hospital admission, but not for those patients who were non ambulant or independently ambulant at hospital admission. This demonstrates the potential for meta-analysis of group mean aggregate data to miss the effect of interventions for subgroups within heterogeneous samples.

Although significant statistical heterogeneity was not identified in the Cochrane review, patient response to the intervention was found to be highly variable across individuals using IPD meta-analysis. Meta regression or sensitivity analysis can be conducted using aggregated patient level characteristics as covariates, but IPD meta-analysis has greater statistical power to detect effects. Very few studies were available to perform either meta-regression or sensitivity analysis in the Cochrane review.

\section{CONCLUSION}

Aggregate systematic review data provides an estimate of an average treatment effect across a group of patients but in clinical practice, results are typically applied to individuals. In heterogeneous patient populations, IPD meta-analysis may provide clinicians with additional information. In this example, performing meta-analysis using group mean data for a heterogeneous older population would have resulted in an intervention that is effective for a subgroup being discarded. This case report supports the recommendations of Tudur Smith et al. [4] who have argued that further comparisons between the outcomes obtained using IPD metaanalysis and group mean aggregate, are required to identify the conditions where IPD meta-analysis may be most beneficial. This report has identified that for reviews investigating the heterogeneous populations, IPD meta-analysis should be recommended to explore the possibility of differential intervention effect across participants.

\section{AUTHOR STATEMENTS}

The authors declare that they have no competing interests.

Both authors were involved in the conception and design of this study, analysis and interpretation of the data, drafting of the manuscript and provided approval of the final version to be published.

\section{FUNDING}

The National Health and Medical Research Council of Australia (Post Doctoral Fellowship, Grant no. 519555).

\section{ROLE OF THE FUNDING SOURCE}

The National Health and Medical Research Council of Australia were not involved in the study design, collection, analysis, interpretation of the data or the writing of this manuscript.

\section{REFERENCES}

[1] Clarke M, Stewart L, Parmer M. Benefits of meta-analysis using individual patient data. Cochrane Colloquia (Abstract) 1995.

[2] Berlin J, Snatanna J, Schmid C, Szczech L, Feldman H. Individual patient vs group-level data meta-regressions for the investigation of treatment effect modifiers: ecological bias rears its ugly head. Stat Med 2002; 21: 371-87.

[3] Lambert P, Sutton A, Jones D. A comparison of summary patientlevel covariates in meta-regression with individual patient data meta-analysis. J Clin Epidemiol 2002; 55: 86-94.

[4] Smith TC, Williamson P, Marson A. An overview of the methods and empirical comparison of aggregate data and individual patient data results for investigating heterogeneity in meta-analysis of time-to-event outcomes. J Eval Clin Pract 2005; 11: 468-78.

[5] de Morton N, Keating J, Jeffs K. The effect of exercise on outcomes for older acute medical inpatients compared to control or alternative treatments: a systematic review of randomised controlled trials. Clin Rehabil 2007; 21:3-16.

[6] de Morton N, Keating J, Jeffs K. Exercise for acutely hospitalised older medical patients. Cochrane Database Syst Rev 2007; (1): CD005955. 
[7] de Morton N, Jones C, Keating J, et al. The effect of exercise on outcomes for hospitalised older acute medical patients: An individual patient data meta-analysis. Age Ageing 2007; 36: 219-22.

[8] Stewart L, Clarke M. Practical methodology of meta-analyses (overviews) using updated individual patient data. Stat Med 1995; 14: 2057-79.

[9] de Morton N, Keating J, Berlowitz D, Jackson B, Lim W. Additional exercise does not change hospital or patient outcomes in older medical patients: a controlled clinical trial. Aust J Physiother 2007; 53: 105-11.

[10] Jones C, Lowe A, Tweddle N, McGregor L, Russell D, Brandt C. A randomised controlled trial of an exercise intervention to reduce functional decline and health service utilization in the hospitalized elderly. Aust J Ageing 2006; 25: 126-33.

(C) de Morton and Keating; Licensee Bentham Open .

This is an open access article licensed under the terms of the Creative Commons Attribution Non-Commercial License (http: //creativecommons.org/licenses/by$\mathrm{nc} / 3.0 /$ ) which permits unrestricted, non-commercial use, distribution and reproduction in any medium, provided the work is properly cited. 